\title{
LIMIT FUNCTIONS OF DISCRETE DYNAMICAL SYSTEMS
}

\author{
H.-P. BEISE, T. MEYRATH, AND J. MÜLLER
}

\begin{abstract}
In the theory of dynamical systems, the notion of $\omega$-limit sets of points is classical. In this paper, the existence of limit functions on subsets of the underlying space is treated. It is shown that in the case of topologically mixing systems on appropriate metric spaces $(X, d)$, the existence of at least one limit function on a compact subset $A$ of $X$ implies the existence of plenty of them on many supersets of $A$. On the other hand, such sets necessarily have to be small in various respects. The results for general discrete systems are applied in the case of Julia sets of rational functions and in particular in the case of the existence of Siegel disks.
\end{abstract}

\section{Limit FUNCTIONS ON SMALL SETS}

Let $(X, d)$ be a complete metric space and let $f: X \rightarrow X$ be continuous. If

$$
f^{\circ n}:=f \circ \cdots \circ f
$$

denotes the $n$-th iterate of $f$ and if $L$ is an arbitrary subset of $X$ we write $\Omega_{p}(L, f)$ for the collection of all functions $g: L \rightarrow X$ that are pointwise limits of some subsequence of $\left(f^{\circ n}\right)_{n}$ on $L$. Necessarily, such functions have to be of Baire class 1 (cf. [8, p. 192]). Moreover, let

$$
\mathcal{K}(X):=\{E \subset X: E \text { nonempty and compact }\} \text {. }
$$

For $E \in \mathcal{K}(X)$, the set of continuous functions from $E$ to $X$ is denoted by $C(E, X)$. We endow $C(E, X)$ with the (complete) uniform metric

$$
d_{u, E}(f, g):=\sup _{x \in E} d(f(x), g(x))
$$

and define $\Omega_{u}(E, f)$ to be the set of all functions that are limits of some subsequence of $\left(f^{\circ n}\right)_{n}$ in $C(E, X)$.

We recall some definitions from topological dynamics. A continuous function $f: X \rightarrow X$ is called topologically transitive if for all nonempty open sets $U, V$ in $X$, an integer $n$ exists which satisfies $f^{\circ n}(U) \cap V \neq \emptyset$. If this holds true for all sufficiently large $n$, then $f$ is called topologically mixing. Finally, $f$ is said to be topologically weak-mixing, if $f \times f$ is topologically transitive on the product space $X \times X$. For basic results on topological transitivity and topological (weak-) mixing we refer to [7. In particular, if $(X, d)$ is separable without isolated points, the Birkhoff transitivity theorem implies that $f$ is topologically weak-mixing if and only if there is a pair $\left(x_{1}, x_{2}\right) \in X \times X$ so that the orbit $\left\{(f \times f)^{\circ n}\left(x_{1}, x_{2}\right): n \in \mathbb{N}\right\}$ is dense in $X \times X$. Thus, $f$ is topologically weak-mixing if and only if there is a

Received by the editors May 22, 2013 and, in revised form, November 20, 2013, December 30, 2013, and December 31, 2013.

2010 Mathematics Subject Classification. Primary 37A25, 37F10, 30 K99.

Key words and phrases. Julia set, limit set, Siegel disk, universality. 
2-point-set $L \subset X$ so that $\Omega_{u}(L, f)=\Omega_{p}(L, f)=C(L, X)=X^{L}(=X \times X)$, that is, the set of limit functions on the 2-point-set $L$ is maximal.

In 1 it is shown that, under certain conditions on the space $X$, topologically weak-mixing functions are universal on many small sets in the sense that the set of limit functions is maximal. In order to formulate the precise result, we introduce some further notations. We set

$$
U_{\rho}(x):=\{y \in X: d(x, y)<\rho\}, \quad U_{\rho}[x]:=\{y \in X: d(x, y) \leq \rho\}
$$

and for $M \subset X$,

$$
U_{\rho}(M):=\bigcup_{x \in M} U_{\rho}(x), \quad U_{\rho}[M]:=\bigcup_{x \in M} U_{\rho}[x] .
$$

On $\mathcal{K}(X)$, the Hausdorff distance is defined by

$$
d_{H}(E, F):=\max \{\delta(E, F), \delta(F, E)\}=\inf \left\{\rho>0: E \subset U_{\rho}[F], F \subset U_{\rho}[E]\right\}
$$

where

$$
\delta(E, F):=\max _{x \in E} \operatorname{dist}(x, F) .
$$

Then $\left(\mathcal{K}(X), d_{H}\right)$ is known to be a complete metric space.

Finally, we say that a property is satisfied for quasi all $x \in X$ (or quasi everywhere) if it is satisfied for all $x$ from a dense $G_{\delta}$ subset of $X$.

With these notations, Akin ([1, Theorem 6.7 and 6.8]) has proved the following result.

Theorem A. Let $(X, d)$ be a complete separable metric space without isolated points and $f: X \rightarrow X$ topologically weak-mixing. Then $\Omega_{u}(E, f)=C(E, X)$ for quasi all $E \in \mathcal{K}(X)$.

Let $\left(Y, d_{Y}\right)$ be a complete metric space. A family $\left\{f_{\iota}: X \rightarrow Y: \iota \in I\right\}$ is called topologically transitive, if, for all $U \subset X$ and $V \subset Y$ open and nonempty, there is $\iota \in I$ with $f_{\iota}(U) \cap V \neq \emptyset$. For $N \in \mathbb{N}$, let $f_{\iota}^{\times N}: X^{N} \rightarrow Y^{N}$ denote the $N$-fold product of $f_{\iota}$. Then $\left\{f_{\iota}^{\times N}: \iota \in I\right\}$ is topologically transitive if, and only if, for all open and nonempty $U_{1}, \ldots, U_{N}$ in $X$ and $V_{1}, \ldots, V_{N}$ in $Y$ there exists $\iota \in I$ with $f_{\iota}\left(U_{j}\right) \cap V_{j} \neq \emptyset$ for all $j=1, \ldots, N$.

Our first result (and the following corollary) will show that, under certain conditions on the spaces $X$ and $Y$, families with $\left\{f_{\iota}^{\times N}: \iota \in I\right\}$ being topologically transitive for all $N$ have "maximal" sets of limit functions on many, but possibly small, compact sets in $X$ similar to those in the case of Theorem A.

Theorem 1.1. Let $X, Y_{0}$ be complete metric spaces so that for a countable set $\left\{h_{k}: k \in \mathbb{N}\right\}$ of continuous functions $h_{k}: X \rightarrow Y_{0}$, the restrictions $\left.h_{k}\right|_{E}$ form a dense set in $C\left(E, Y_{0}\right)$ for quasi all $E \in \mathcal{K}(X)$. Moreover, let $Y \subset Y_{0}$ be closed and suppose that $f_{\iota}: X \rightarrow Y$ are continuous for every $\iota \in I$ and that $\left\{f_{\iota}^{\times N}: \iota \in I\right\}$ is topologically transitive for all $N \in \mathbb{N}$. Then $\left\{\left.f_{\iota}\right|_{E}: \iota \in I\right\}$ is dense in $C(E, Y)$ for quasi all $E \in \mathcal{K}(X)$.

Proof. For $k, m \in \mathbb{N}$ we define $\mathcal{B}_{k, m}$ to be the set of all $M \in \mathcal{K}(X)$ so that $d_{u, M}\left(h_{k}, f_{\iota}\right)<\delta\left(h_{k}(M), Y\right)+\frac{1}{m}$ for some $\iota \in I$.

We show that $\mathcal{B}_{k, m}$ is open and dense in $\mathcal{K}(X)$ for every choice of $k, m \in \mathbb{N}$. For that purpose, let $k, m \in \mathbb{N}, M \in \mathcal{K}(X)$ be fixed and assume that $\iota \in I$ satisfies $d_{u, M}\left(h_{k}, f_{\iota}\right)<\delta\left(h_{k}(M), Y\right)+1 / m$. By the continuity of $f_{\iota}$ and $h_{k}$, the existence 
of a Lebesgue number for all open coverings of $M$ implies that there is a $\rho>0$ so that

$$
d\left(h_{k}(x), f_{\iota}(x)\right)<\delta\left(h_{k}(M), Y\right)+\frac{1}{m} \quad \text { for all } x \in U_{\rho}[M] .
$$

From this, one verifies that $\left\{K \in \mathcal{K}(X): d_{H}(K, M)<\rho\right\}$ is an open neighbourhood of $M$ that is contained in $\mathcal{B}_{k, m}$, which proves that $\mathcal{B}_{k, m}$ is open.

In order to show the denseness of $\mathcal{B}_{k, m}$, let $k, m \in \mathbb{N}, K \in \mathcal{K}(X)$, and $\varepsilon>0$ be fixed. For each $x \in X$, let $\varepsilon>\delta_{x}>0$ be such that $d\left(h_{k}(x), h_{k}(\tilde{x})\right)<1 / 3 m$ whenever $d(x, \tilde{x})<\delta_{x}$. Then due to the compactness of $K$, there is a finite set $F:=\left\{x_{1}, \ldots, x_{N}\right\} \subset K$ such that $K \subset \bigcup_{j=1}^{N} U_{j}$, where $U_{j}:=U_{\delta_{x_{j}}}\left(x_{j}\right)$. We further define

$$
V_{j}:=\left\{y \in Y: \operatorname{dist}\left(y, h_{k}\left(U_{j}\right)\right)<\operatorname{dist}\left(h_{k}\left(U_{j}\right), Y\right)+1 / 3 m\right\}(\neq \emptyset)
$$

for $j=1, \ldots, N$. Now, the assumption on $\left(f_{\iota}\right)$ implies that there is a $\iota \in I$ so that $f_{\iota}\left(U_{j}\right) \cap V_{j} \neq \emptyset$ for every $j=1, \ldots, N$.

For every $j$, we may take a $w_{j} \in U_{j}$ so that $f_{\iota}\left(w_{j}\right) \in V_{j}$ and, by the definition of $V_{j}$, we find a corresponding $u_{j} \in U_{j}$ that satisfies $d\left(f_{\iota}\left(w_{j}\right), h_{k}\left(u_{j}\right)\right)<$ $\operatorname{dist}\left(h_{k}\left(U_{j}\right), Y\right)+1 / 3 m$. Together with the definition of the sets $U_{j}$, we obtain

$$
\begin{aligned}
d\left(f_{\iota}\left(w_{j}\right), h_{k}\left(w_{j}\right)\right) & \leq d\left(f_{\iota}\left(w_{j}\right), h_{k}\left(u_{j}\right)\right)+d\left(h_{k}\left(u_{j}\right), h_{k}\left(x_{j}\right)\right) \\
& +d\left(h_{k}\left(x_{j}\right), h_{k}\left(w_{j}\right)\right) \\
& <\operatorname{dist}\left(h_{k}\left(U_{j}\right), Y\right)+\frac{1}{m} \\
& \leq \operatorname{dist}\left(h_{k}\left(w_{j}\right), Y\right)+\frac{1}{m}
\end{aligned}
$$

for all $j=1, \ldots, N$. This shows that $M:=\left\{w_{1}, \ldots, w_{N}\right\} \in \mathcal{B}_{k, m}$. Since the $\delta_{x_{j}}$ are chosen to be less than $\varepsilon$, we have $d_{H}(M, K)<\varepsilon$ and since $\varepsilon>0$ was arbitrary, this proves the denseness of $\mathcal{B}_{k, m}$ in $\mathcal{K}(X)$.

According to Baire's theorem, the set $\bigcap_{k, m \in \mathbb{N}} \mathcal{B}_{k, m}$ is a dense $G_{\delta}$ set in $\mathcal{K}(X)$. Thus, it suffices to show that each $E$ in this intersection has the claimed property. To this aim, let $g \in C(E, Y)$ and let $\varepsilon>0$. We choose $k, m$ so that $1 / m<\varepsilon / 3$ and $d_{u, E}\left(g, h_{k}\right)<\varepsilon / 3$. Since $g(E) \subset Y$ we have $\delta\left(h_{k}(E), Y\right)<\varepsilon / 3$ and since $E$ belongs to $\mathcal{B}_{k, m}$ we obtain

$$
d_{u, E}\left(g, f_{\iota}\right) \leq d_{u, E}\left(g, h_{k}\right)+d_{u, E}\left(h_{k}, f_{\iota}\right)<\varepsilon / 3+\delta\left(h_{k}(E), Y\right)+1 / m<\varepsilon
$$

for some $\iota \in I$.

Let $p, q \in \mathbb{N}$. If $Y_{0}=\mathbb{K}^{q}$ and $X \subset \mathbb{K}^{p}$ is closed, then a sequence $\left(h_{k}\right)$ as in Theorem 1.1 exists. Indeed, according to the Stone-Weierstrass theorem (see e.g. $[18$, p. 115]), the polynomials in $p($ case $\mathbb{K}=\mathbb{R})$ or $2 p($ case $\mathbb{K}=\mathbb{C}$ ) real variables with rational (or Gaussian rational) coefficients form a countable set which is dense in $C(E, \mathbb{K})$ for all compact $E \subset X$. Thus, the corresponding vector-valued functions constitute a sequence $\left(h_{k}\right)$ as desired. From Theorem 1.1 we get

Corollary 1.2. Let $X \subset \mathbb{K}^{p}$ and $Y \subset \mathbb{K}^{q}$ be closed and suppose $f_{\iota}: X \rightarrow Y(\iota \in I)$ to be continuous functions with the property that $\left\{f_{\iota}^{\times N}: \iota \in I\right\}$ is topologically transitive for all $N \in \mathbb{N}$. Then $\left\{\left.f_{\iota}\right|_{E}: \iota \in I\right\}$ is dense in $C(E, Y)$ for quasi all $E \in \mathcal{K}(X)$. 
Example 1.3. Let $f \in C(\mathbb{R}, \mathbb{C})$ be periodic and satisfy $f(\mathbb{R})=Y$. Furthermore, let $X \subset \mathbb{R}$ be a closed interval containing more than one point. For a sequence of real numbers $\left(\alpha_{n}\right)$ tending to $\infty$ we consider the functions $f_{n}: X \rightarrow Y$ defined by

$$
f_{n}(x):=f\left(\alpha_{n} x\right) \quad(x \in X) .
$$

Then, for arbitrary open nonempty $U \subset X$, we have $f_{n}(U)=Y$ for $n$ sufficiently large, so that the assumptions of Corollary 1.2 are satisfied. Thus, $\left\{f_{n}: n \in \mathbb{N}\right\}$ is dense in $C(E, Y)$ for quasi all $E \in \mathcal{K}(X)$.

For example, if we consider $f(x)=\sin (x)$ and $f_{n}(x)=\sin \left(\alpha_{n} x\right)$, we obtain that $\left\{f_{n}: n \in \mathbb{N}\right\}$ is dense in $C(E,[-1,1])$ for quasi all $E \in \mathcal{K}(X)$. A further example is given by $f(x):=e^{i x}$ and $f_{n}(x)=e^{i \alpha_{n} x}$. Applying the above yields that $\left\{f_{n}: n \in \mathbb{N}\right\}$ is dense in $C(E, \mathbb{T})$ for quasi all $E \in \mathcal{K}(X)$, where $\mathbb{T}$ denotes the unit circle in $\mathbb{C}$.

Remark 1.4. Let $X$ and $f$ be as in Theorem $\mathrm{A}$. Then according to Furstenberg's theorem (see, e.g. [7, Theorem 1.51]), the family $\left\{\left(f^{\circ n}\right)^{\times N}: X^{N} \rightarrow X^{N}: n \in \mathbb{N}\right\}$ is topologically transitve for all $N \in \mathbb{N}$. Therefore, Corollary 1.2 recovers the statement of Theorem $\mathrm{A}$ in the special case that $X \subset \mathbb{K}^{p}$ for some $p \in \mathbb{N}$.

We show how Corollary 1.2 can be further applied in dynamics. Let $X \subset \mathbb{K}^{p}$ be closed and $A \subset X$ compact. For $H$ closed in $C(A, X)$ and $E \in \mathcal{K}(X)$ we define

$$
C_{H}(E \cup A, X):=\left\{g \in C(E \cup A, X):\left.g\right|_{A} \in H\right\} .
$$

Theorem 1.5. Let $X$ be a closed subset of $\mathbb{K}^{p}$ without isolated points, and suppose that $f: X \rightarrow X$ is continuous and topologically mixing. If $A \in \mathcal{K}(X)$ and $H:=$ $\Omega_{u}(A, f) \neq \emptyset$, then $C_{H}(E \cup A, X)=\Omega_{u}(E \cup A, f)$ for quasi all $E$ in $\mathcal{K}(X)$.

Proof. From the fact that $f$ is topologically mixing, it is easily seen that every subsequence $\left(f_{j}:=f^{\circ n_{j}}\right)_{j \in \mathbb{N}}$ of $\left(f^{\circ n}\right)_{n \in \mathbb{N}}$ satisfies the conditions of Corollary 1.2 (with $Y=X$ ).

Moreover, as above, the Stone-Weierstrass theorem shows that the Fréchet space $C\left(\mathbb{K}^{p}, \mathbb{K}^{p}\right)$ endowed with the topology of locally uniform convergence is separable. Therefore, also the closed subset

$$
C_{H}\left(\mathbb{K}^{p}, \mathbb{K}^{p}\right):=\left\{g \in C\left(\mathbb{K}^{p}, \mathbb{K}^{p}\right):\left.g\right|_{A} \in H\right\}
$$

is separable. Let $\left\{h_{k}: k \in \mathbb{N}\right\}$ be dense in $C_{H}\left(\mathbb{K}^{p}, \mathbb{K}^{p}\right)$. Then Tietze's extension theorem (see e.g. [19, Theorem 20.4]) implies that $\left\{\left.h_{k}\right|_{E \cup A}: k \in \mathbb{N}\right\}$ is dense in $C_{H}\left(E \cup A, \mathbb{K}^{p}\right)$ for quasi all $E \in \mathcal{K}(X)$.

Since $h_{k} \in C_{H}\left(\mathbb{K}^{p}, \mathbb{K}^{p}\right)$ and thus $\left.h_{k}\right|_{A} \in H$ for all $k \in \mathbb{N}$, there exists a subsequence $\left(f_{j}:=f^{\circ n_{j}}\right)_{j \in \mathbb{N}}$ of $\left(f^{\circ n}\right)_{n \in \mathbb{N}}$ with $d_{u, A}\left(f_{j}, h_{j}\right)<1 / j$ for all $j \in \mathbb{N}$. Since $X$ has no isolated points, Corollary 1.2 implies that $\left\{f_{j}: j \geq j_{0}\right\}$ is dense in $C(E, X)$ for quasi all $E \in \mathcal{K}(X)$ and all $j_{0} \in \mathbb{N}$. If $g \in C_{H}(E \cup A, X)$ and $\varepsilon>0$ are given, there is $j \in \mathbb{N}$ with $1 / j<\varepsilon / 2, d_{u, A}\left(h_{j}, g\right)<\varepsilon / 2$ and $d_{u, E}\left(f_{j}, g\right)<\varepsilon$. This implies $d_{u, E \cup A}\left(g, f_{j}\right)<\varepsilon$.

Theorem 1.5 shows that, under the corresponding conditions, the existence of at least one limit function on a set $A$ implies the existence of plenty of them on many sets containing $A$. In the next section we will give an example where the set $A$ is nontrivial.

In the opposite direction, the following result shows that sets with $\Omega_{p}(L, f) \neq \emptyset$ have to be topologically small. 
Proposition 1.6. Let $(X, d)$ be a complete metric space so that $|X| \geq 2$ and let $f: X \rightarrow X$ be continuous and topologically mixing. Then $\Omega_{p}(L, f)=\emptyset$ for all $L \subset X$ of the second category.

Proof. For two elements $x \neq y$ of $X$ and $d(x, y)=a$ we define $U:=U_{a / 4}(x)$ and $V:=U_{a / 4}(y)$. Assume that $f^{\circ n_{j}} \rightarrow h$ is pointwise on $L$ for some function $h: L \rightarrow X$ and some increasing sequence of positive integers $\left(n_{j}\right)$. We have to show that $L$ is of the first category.

Since $f$ is mixing, we have that $U_{m}:=\bigcup_{j>m}\left(f^{\circ n_{j}}\right)^{-1}(U)$ and $V_{m}:=$ $\bigcup_{j>m}\left(f^{\circ n_{j}}\right)^{-1}(V)$ are open and dense in $X$ for every positive integer $m$. Baire's theorem implies that $M:=\bigcap_{m \in \mathbb{N}}\left(U_{m} \cap V_{m}\right)$ is a dense $\mathrm{G}_{\delta}$-set. From the fact that $d(u, w)>a / 2>0$ for all $u \in U, w \in V$ we obtain that $L \subset X \backslash M$.

If an (invariant) Borel measure on $X$ exists, we can ask whether limit functions may appear on sets of positive or even full measure.

The following result shows that in the case of existence of a probability measure $\mu$ on the Borel sets of $X$ so that $f$ is (metrically) strong-mixing, pointwise limit functions exist only on sets of zero $\mu$-measure. We recall that a measure-preserving transformation $f: X \rightarrow X$ is called (metrically) strong-mixing, if

$$
\lim _{n \rightarrow \infty} \mu\left(\left(f^{\circ n}\right)^{-1}(A) \cap B\right)=\mu(A) \mu(B)
$$

for all Borel sets $A, B$. For further notions from ergodic theory we refer to [22].

Theorem 1.7. Let $X$ with $|X| \geq 2$ be a metric space possessing a compact exhaustion and let $\mu$ be a Borel probability measure on $X$ not being a Dirac measure. If $f: X \rightarrow X$ is continuous, $\mu$-invariant and strong-mixing with respect to $\mu$, then $\Omega_{p}(L, f)=\emptyset$ for all Borel sets $L$ with $\mu(L)>0$.

Proof. Let a Borel set $L$ of positive $\mu$-measure be given and suppose that there exists a sequence $\left(f^{\circ n_{j}}\right)_{j \in \mathbb{N}}$ tending pointwise to some (Baire) function $h$ on $L$. According to Egoroff's theorem, the convergence is uniform on a subset $E$ of $L$ again having positive $\mu$-measure. The inner regularity of $\mu$ (see [16, Lemma A. 3.3]) shows that $E$ can be chosen to be compact. Thus, it suffices to show that $\Omega_{u}(E, f)=\emptyset$ for all compact subsets $E$ of $X$ with $\mu(E)>0$.

Consider $E$ to be compact in $X$ with $\mu(E)>0$.

1. We show that for each $h \in \Omega_{u}(E, f)$ the image $h(A)$ has full $\mu$-measure for all closed subsets $A$ of $E$ with $\mu(A)>0$.

Indeed: Suppose that $f^{\circ n_{j}} \rightarrow h$ uniformly on $E$ as $j \rightarrow \infty$. Let $A$ be a closed subset of $E$ with positive measure. Then, for each $\delta>0$ there is a $j_{\delta}$ so that

$$
A \subset\left(f^{\circ n_{j}}\right)^{-1}\left(U_{\delta}(h(A))\right)
$$

for all $j \geq j_{\delta}$. From the strong-mixing property of $f$ we obtain

$$
\mu(A)=\mu\left(A \cap\left(f^{\circ n_{j}}\right)^{-1}\left(U_{\delta}(h(A))\right)\right) \rightarrow \mu(A) \mu\left(U_{\delta}(h(A))\right) \quad(j \rightarrow \infty) .
$$

Since $\mu(A)$ is positive, this implies $\mu\left(U_{\delta}(h(A))\right)=1$ and since $\delta>0$ was arbitrary, the continuity of $\mu$ from above implies

$$
\mu(\overline{h(A)})=\lim _{k \rightarrow \infty} \mu\left(U_{1 / k}(h(A))\right)=1 .
$$

Moreover, the limit function $h$ is continuous and therefore $h(A)$ is compact in $X$ and thus $\overline{h(A)}=h(A)$. 
2. The compactness of $E$ implies the existence of a point $x \in E$ so that $\mu(E \cap$ $\left.U_{\delta}(x)\right)>0$ for all positive $\delta$. Let $\varepsilon>0$ be so small that $\mu\left(U_{\varepsilon}(h(x))\right)<1$ (such an $\varepsilon$ exists since $\mu$ is not the Dirac measure at $h(x))$. Then there is a positive $\delta$ so that

$$
h\left(U_{\delta}[x] \cap E\right) \subset U_{\varepsilon}(h(x)) .
$$

Considering $A=U_{\delta}[x] \cap E$ in 1 . we obtain the contradicting statement

$$
1>\mu\left(U_{\varepsilon}(h(x))\right) \geq \mu(h(A))=1 .
$$

Remark 1.8. If $g: \mathbb{T} \rightarrow \mathbb{T}$ is an irrational rotation, i.e., $g(z)=\alpha z(z \in \mathbb{T})$, where $\alpha \in \mathbb{T}$ is not a root of unity, it is easily seen that $h \in \Omega_{u}(E, g)$ for an arbitrary closed subset $E$ of $\mathbb{T}$ if and only if $h$ is of the form $h(z)=\beta z$ for some $\beta \in \mathbb{T}$. Thus, uniform limit functions exist on the full space $X=\mathbb{T}$. Since irrational rotations are ergodic, this shows that the assertion of Theorem 1.7 no longer holds for ergodic mappings in general.

Even in the case of the mere existence of an invariant measure, there is a restriction on possible limit functions:

Proposition 1.9. Let $X \subset \mathbb{K}^{p}$ be closed, $\mu$ a Radon measure on $X$, and $f: X \rightarrow X$ $\mu$-invariant. If $L \subset X$ is a Borel set and if $h \in \Omega_{p}(L, f)$, then $\mu(L) \leq \mu(\overline{h(L)})$. In particular, if $\mu$ has no point mass and $h$ is constant, then $\mu(L)=0$.

Proof. As in the proof of Theorem 1.7. from Egoroff's theorem and the inner regularity of $\mu$ it follows that it is sufficient to prove the result for $h \in \Omega_{u}(E, f)$, where $E \subset L$ is compact.

If $f^{\circ n_{j}} \rightarrow h$ uniformly on $E$, then for all $\delta>0$,

$$
E \subset\left(f^{\circ n_{j}}\right)^{-1}\left(U_{\delta}(h(E))\right)
$$

for $j$ sufficiently large. Thus

$$
\mu(E) \leq \mu\left(\left(f^{\circ n_{j}}\right)^{-1}\left(U_{\delta}(h(E))\right)\right)=\mu\left(U_{\delta}(h(E))\right)
$$

for $j$ sufficiently large. Since $\delta>0$ was arbitrary, and since $\mu\left(U_{\delta}(h(E))\right)<\infty$ (note that $h(E)$ is compact and therefore $U_{\delta}[h(E)]$ also), continuity from above shows $\mu(L) \leq \mu(\overline{h(L)})$.

In the next section, we give applications in one-dimensional complex dynamics.

\section{Applications in COMPlex Dynamics}

Let $f$ be an entire function, not a polynomial of degree 0 or 1 . The Julia set $J=J(f)$ of $f$ is defined as the set of all $z \in \mathbb{C}$ so that the iterates $f^{\circ n}$ do not form a (spherically) normal family in any open neighborhood of $z$. For properties of the Julia set of an entire function we refer to the expository articles [3] and [20]. In particular, $J$ is compact if $f$ is a polynomial, and is closed and unbounded in $\mathbb{C}$ if $f$ is transcendental. Moreover, $J$ has no isolated points.

In the case of a polynomial $f$, it is well known that, for all relatively open sets $U$ in $J$,

$$
f^{\circ n}(U)=J
$$

for all $n$ sufficiently large (see e.g. [5, Theorem 3.2], [14, Corollary 14.2], [21, p. 36]). Thus, polynomials are topologically mixing on their Julia set. If $f$ is a transcendental entire function, then it can be shown that $f$ is still topologically weak-mixing 
(cf. the considerations in [2]). Thus, Theorem $\mathrm{A}$ shows that $\Omega_{u}(E, f)=C(E, J)$ for quasi all $E \in \mathcal{K}(J)$. This gives another proof of the theorem from [2].

In the case of rational functions, the Julia set has to be considered in the extended plane $\mathbb{C}_{\infty}:=\mathbb{C} \cup\{\infty\}$ which we endow with the chordal metric $\chi$. Again, rational functions are topologically mixing on their Julia set (see e.g. [5, Theorem 3.2], [14. Corollary 14.2]) and the Julia set is perfect.

As a consequence of Theorem A, we obtain the following result similar to the theorem from [2].

Theorem 2.1. Let $f$ be a rational function of degree $\geq 2$ with Julia set $J$. Then for quasi all $E \in \mathcal{K}(J)$ we have $\Omega_{u}(E, f)=C(E, J)$.

In [2] it is shown that in the case of dynamics of entire functions $f$, pointwise limit functions only exist on sets that are topologically small. More precisely, if $L$ is of the second category in the Julia set $J=J(f)$, then $\Omega_{p}(L, f)=\emptyset$. For polynomials (and more generally for rational functions) this is now also a consequence of Proposition 1.6 .

Our aim is to consider a typical situation in which Theorem 1.5 may be applied.

Let $f$ be a rational function with Julia set $J$ in $\mathbb{C}$ and having a Siegel disk $F$ with fixed point $w_{0} \in \mathbb{C}_{\infty}$ and so that the boundary $\partial F$ of $F$ is a Jordan curve. Then each inverse Riemann mapping $\varphi: \mathbb{D} \rightarrow F$ with $\varphi(0)=w_{0} \in F$ induces a homeomorphism (also called $\varphi$ ) from $\mathbb{T}$ to $\partial F$. Since $\varphi: \mathbb{T} \rightarrow \partial F$ is a homeomorphism that conjugates $f$ with $g=g_{\alpha}$, where $g$ is an irrational rotation (see, e.g. [5, Theorem II 6.4] or [21, pp. 80]) we obtain from Remark 1.8 that $h \in \Omega_{u}(E, f)$ for some (and then all) $E \in \mathcal{K}(\partial F)$ if and only if

$$
h=\varphi \circ\left(\beta \cdot \operatorname{id}_{\mathbb{T}}\right) \circ \varphi^{-1}
$$

for some $\beta \in \mathbb{T}$. Theorem 2.1 implies that quasi all $E \in \mathcal{K}(J)$ do not intersect $\partial F$ in more than one point.

Denoting by $H$ the set of all functions $h$ of the above form (11), Theorem 1.5. applied with $X=J$ and $A=\partial F$, shows that the following result holds.

Theorem 2.2. Let $f$ be a rational function with Julia set in $\mathbb{C}$ and having a Siegel disk $F$ with Jordan boundary $\partial F$. Then

$$
\Omega_{u}(E \cup \partial F, f)=C_{H}(E \cup \partial F, J)
$$

for quasi all $E \in \mathcal{K}(J)$.

We consider the situation from the measure-theoretic point of view. For each rational function $f$ of degree at least 2 there exists a probability measure $\mu$ whose support is $J$ so that $f$ is $\mu$-invariant and strong-mixing ([6], [1] ). In the case of a polynomial $f$, this measure coincides with the equilibrium measure $\nu$ of the Julia set (see [4] see also [15]). From Theorem 1.7 we get

Corollary 2.3. Let $f$ be a rational function of degree $\geq 2$. Then $\Omega_{p}(L, f)=\emptyset$ for all Borel sets $L \subset J$ with $\mu(L)>0$.

Remark 2.4. Again, let $f$ be a rational function of degree at least 2. Even if $\infty$ is a fixed point belonging to the Fatou set, which implies that $J$ is a compact subset of the plane with positive capacity and having the property that $f$ is invariant with respect to the equilibrium measure $\nu$ of $J$ (see [9, Theorem 1]), the maximal entropy measure $\mu$ is different from the equilibrium measure $\nu$ unless $f$ is a polynomial (see 
[10], and [15] for an extension). In particular, if $\infty$ is a neutral fixed point, then the system $(J, f, \nu)$ is a factor of an irrational rotation of the circle, hence ergodic with respect to $\nu$, and $\nu$ and $\mu$ are mutually singular (again, see [9, Theorem 1]). In this case, the component $F_{\infty}$ of the Fatou set containing $\infty$ is a Siegel disk (see [9, Theorem 1 and the corresponding proof]).

We show that in the case of a Siegel disk with Jordan boundary, limit functions on sets of full $\nu$-measure exist: Since the support of $\nu$ is in $\partial F_{\infty}, \partial F_{\infty}$ has full $\nu$-measure. We obtain from Theorem 2.2 that

$$
C_{H}\left(E \cup \partial F_{\infty}, J\right)=\Omega_{u}\left(E \cup \partial F_{\infty}, f\right)
$$

for quasi all $E \in \mathcal{K}(J)$.

Since $\mu$ and $\nu$ are mutually singular, $\partial F_{\infty}$ is a $\mu$-null set. This shows that the behavior concerning limit functions appears fundamentally different depending on which underlying invariant measure is considered.

Remark 2.5. For the exponential function $\exp : \mathbb{C} \rightarrow \mathbb{C}$ we have $J(\exp )=\mathbb{C}$. It is known that no absolutely continuous Radon measure $\mu$ on the Borel sets in $\mathbb{C}$ exists so that $\exp$ is $\mu$-invariant (see [13, Theorem 1.3]). In view of Proposition 1.9 it would be interesting to know if there is a constant limit function $h$ on a set of positive planar Lebesgue measure. In any case, such a limit function $h$ can take its value only in the postsingular set $\left\{\exp ^{\circ n}(0): n \in \mathbb{N}_{0}\right\}$, as follows from [12] or [17].

As already mentioned above, in [2, Proposition 1] it is shown that (also) in the case of entire functions $f$, pointwise limit functions can only exist on sets of the first category in $J$. The proof of the result shows that $g \equiv \infty$ also cannot appear as a limit function on a set of the second category. On the other hand, $g \equiv \infty$ may be a limit function on sets of full planar Lebesgue measure, as for instance in the case of postcritically finite maps in the cosine family, where $J=\mathbb{C}$ and where the escaping set has full measure in the sense that the complement has measure zero (see, e.g. [20, Theorem 5.4 and p. 324]).

\section{ACKNOWLEDGMENT}

We would like to thank the anonymous referee for his thorough review and his insightful comments that helped us to improve the original version of the manuscript.

\section{REFERENCES}

[1] Ethan Akin, Lectures on Cantor and Mycielski sets for dynamical systems, Chapel Hill Ergodic Theory Workshops, Contemp. Math., vol. 356, Amer. Math. Soc., Providence, RI, 2004, pp. 21-79, DOI 10.1090/conm/356/06496. MR2087588(2005e:37018)

[2] Peter Beise and Jürgen Müller, Limit functions of iterates of entire functions on parts of the Julia set, Proc. Amer. Math. Soc. 141 (2013), no. 11, 3929-3933, DOI 10.1090/S0002-99392013-11720-X. MR3091783

[3] Walter Bergweiler, Iteration of meromorphic functions, Bull. Amer. Math. Soc. (N.S.) 29 (1993), no. 2, 151-188, DOI 10.1090/S0273-0979-1993-00432-4. MR.1216719 (94c:30033)

[4] Hans Brolin, Invariant sets under iteration of rational functions, Ark. Mat. 6 (1965), 103-144 (1965). MR0194595(33 \#2805)

[5] Lennart Carleson and Theodore W. Gamelin, Complex dynamics, Universitext: Tracts in Mathematics, Springer-Verlag, New York, 1993. MR.1230383 (94h:30033)

[6] Alexandre Freire, Artur Lopes, and Ricardo Mañé, An invariant measure for rational maps, Bol. Soc. Brasil. Mat. 14 (1983), no. 1, 45-62, DOI 10.1007/BF02584744. MR736568 (85m:58110b) 
[7] Karl-G. Grosse-Erdmann and Alfredo Peris Manguillot, Linear chaos, Universitext, Springer, London, 2011. MR2919812

[8] Alexander S. Kechris, Classical descriptive set theory, Graduate Texts in Mathematics, vol. 156, Springer-Verlag, New York, 1995. MR1321597(96e:03057)

[9] Steven P. Lalley, Brownian motion and the equilibrium measure on the Julia set of a rational mapping, Ann. Probab. 20 (1992), no. 4, 1932-1967. MR1188049 (94f:58079)

[10] Artur Oscar Lopes, Equilibrium measures for rational maps, Ergodic Theory Dynam. Systems 6 (1986), no. 3, 393-399, DOI 10.1017/S0143385700003576. MR863202(88e:58055)

[11] M. Ju. Ljubich, Entropy properties of rational endomorphisms of the Riemann sphere, Ergodic Theory Dynam. Systems 3 (1983), no. 3, 351-385, DOI 10.1017/S0143385700002030. MR:741393 (85k:58049)

[12] M. Yu. Lyubich, Generic behavior of trajectories of the exponential function (Russian), Uspekhi Mat. Nauk 41 (1986), no. 2(248), 199-200. MR.842176 (87g:58062)

[13] M. Yu. Lyubich, The measurable dynamics of the exponential (Russian), Sibirsk. Mat. Zh. 28 (1987), no. 5, 111-127. MR924986 (89d:58071)

[14] John Milnor, Dynamics in one complex variable, Friedr. Vieweg \& Sohn, Braunschweig, 1999. Introductory lectures. MR 1721240 (2002i:37057)

[15] Yûsuke Okuyama and Małgorzata Stawiska, Potential theory and a characterization of polynomials in complex dynamics, Conform. Geom. Dyn. 15 (2011), 152-159, DOI 10.1090/S10884173-2011-00230-X. MR2846305

[16] Thomas Ransford, Potential theory in the complex plane, London Mathematical Society Student Texts, vol. 28, Cambridge University Press, Cambridge, 1995. MR1334766 (96e:31001)

[17] Mary Rees, The exponential map is not recurrent, Math. Z. 191 (1986), no. 4, 593-598, DOI 10.1007/BF01162349. MR832817 (87g:58063)

[18] Walter Rudin, Functional analysis, McGraw-Hill Book Co., New York, 1973. McGraw-Hill Series in Higher Mathematics. MR0365062 (51 \#1315)

[19] Walter Rudin, Real and complex analysis, 3rd ed., McGraw-Hill Book Co., New York, 1987. MR.924157 (88k:00002)

[20] Dierk Schleicher, Dynamics of entire functions, Holomorphic dynamical systems, Lecture Notes in Math., vol. 1998, Springer, Berlin, 2010, pp. 295-339, DOI 10.1007/978-3-642-131714_5. MR2648691 (2011h:37070)

[21] Norbert Steinmetz, Rational iteration, de Gruyter Studies in Mathematics, vol. 16, Walter de Gruyter \& Co., Berlin, 1993. Complex analytic dynamical systems. MR.1224235 (94h:30035)

[22] Peter Walters, An introduction to ergodic theory, Graduate Texts in Mathematics, vol. 79, Springer-Verlag, New York, 1982. MR648108(84e:28017)

Department of Mathematics, University of Trier, 54286 Trier, Germany

E-mail address: pbeise@gmx.de

University of Luxembourg, Faculte des Sciences 6, Rue Richard Coudenhove-Kalergi, L-1359, LuXEMBOURG

E-mail address: thierry.meyrath@uni.lu

University of Trier, Mathematik, Fachbereich IV, 54286 Trier, Germany

E-mail address: jmueller@uni-trier.de 